Article

\title{
Several Results of Fractional Differential and Integral Equations in Distribution
}

\author{
Chenkuan $\mathrm{Li}^{1, *}$, Changpin $\mathrm{Li}^{2}$ and Kyle Clarkson ${ }^{1}$ \\ 1 Department of Mathematics and Computer Science, Brandon University, Brandon, MB R7A 6A9, Canada; \\ kyleclarkson17@hotmail.com \\ 2 Department of Mathematics, Shanghai University, Shanghai 200444, China; lcp@shu.edu.cn \\ * Correspondence: lic@brandonu.ca
}

Received: 10 May 2018; Accepted: 6 June 2018; Published: 8 June 2018

Abstract: This paper is to study certain types of fractional differential and integral equations, such as $\theta\left(x-x_{0}\right) g(x)=\frac{1}{\Gamma(\alpha)} \int_{0}^{x}(x-\zeta)^{\alpha-1} f(\zeta) d \zeta, y(x)+\int_{0}^{x} \frac{y(\tau)}{\sqrt{x-\tau}} d \tau=x_{+}^{-\sqrt{2}}+\delta(x)$, and $x_{+}^{k} \int_{0}^{x} y(\tau)(x-$ $\tau)^{\alpha-1} d \tau=\delta^{(m)}(x)$ in the distributional sense by Babenko's approach and fractional calculus. Applying convolutions and products of distributions in the Schwartz sense, we obtain generalized solutions for integral and differential equations of fractional order by using the Mittag-Leffler function, which cannot be achieved in the classical sense including numerical analysis methods, or by the Laplace transform.

Keywords: distribution; fractional calculus; convolution; Abel's integral equation; product; Mittag-Leffler function

MSC: 46F10; 26A33; 45E10; 45G05

\section{Introduction}

Fractional calculus deals with integrals and derivatives of arbitrary order, which unifies and extends integer-order differentiation and $n$-fold integration. Up to now, fractional operators have been applied in various areas, such as anomalous diffusion, long-range interactions, long-memory processes and materials, waves in liquids, and physics. Recently, Zimbardo et al. [1] generalized the Parker equation, which describes the acceleration and transport of energetic particles in astrophysical plasmas, to the case of anomalous by Caputo fractional derivatives. As far as we know, fractional calculus is one of best tools to construct certain electro-chemical problems and characterizes long-term behaviors, allometric scaling laws, nonlinear operations of distributions [2] and so on.

An integral equation is an equation containing an unknown function under an integral sign. Integral equations are useful and powerful mathematical tools in both pure and applied mathematics. They have various applications in numerous physical problems, chemistry, biology, electronics and mechanics [3-5]. Many initial and boundary value problems associated with ordinary (or partial) differential equations can be transformed into problems of solving integral equations [6]. For example, Gorenflo and Mainardi [7] provided interesting applications of Abel's integral equations of the first and second kind in solving the partial differential equation which describes the problem of the heating (or cooling) of a semi-infinite rod by influx (or efflux) of heat across the boundary into (or from) its interior. There have been lots of approaches, including numerical analysis, thus far to studying fractional differential and integral equations, including Abel's equations, with many applications [8-21]. Recently, Li et al. [22,23] studied integral equations associated with Abel's types in the distributional (Schwartz) sense, based on new fractional calculus of distributions and derived fresh results which are not achievable in the classical sense. On the other hand, the development of science has led to 
formation of many physical and engineering problems that can be mathematically represented by differential equations. For instance, problems from electric circuits, chemical kinetics, and transfer of heat can all be characterized as differential equations [24].

We briefly introduce the necessary concepts and definitions of fractional calculus of distributions in $\mathcal{D}^{\prime}\left(R^{+}\right)$in Section 2, where we demonstrate several examples of computing fractional derivatives as well as applications to solving Abel's integral equations of the first kind for arbitrary $\alpha \in R$. In Section 3, we present Babenko's approach to solving several fractional differential and integral equations based on new convolution and product of generalized functions (an active area in distribution theory). We often obtain an infinite series, related to the Mittag-Leffler function, as the solution of integral or differential equation. We imply a number of novel results, which cannot be derived or approximated by numerical analysis methods or by the Laplace transform, since solutions are in the distributional sense in general.

\section{Fractional Calculus in $\mathcal{D}^{\prime}\left(R^{+}\right)$}

In order to investigate fractional integral and differential equations in the generalized sense, we briefly introduce the following basic concepts, with several interesting examples of solving Abel's integral equations in distribution. Let $\mathcal{D}(R)$ be the Schwartz space (testing function space) [25] of infinitely differentiable functions with compact support in $R$, and $\mathcal{D}^{\prime}(R)$ the (dual) space of distributions defined on $\mathcal{D}(R)$. A sequence $\phi_{1}, \phi_{2}, \cdots, \phi_{n}, \cdots$ goes to zero in $\mathcal{D}(R)$, if and only if these functions vanish outside a certain fixed bounded set, and converge to zero uniformly together with their derivatives of any order. Clearly, $\mathcal{D}(R)$ is not empty since it contains the following function

$$
\phi(x)= \begin{cases}e^{-\frac{1}{1-x^{2}}} & \text { if }|x|<1 \\ 0 & \text { otherwise }\end{cases}
$$

Evidently, any locally integrable function $f(x)$ on $R$ is a (regular) distribution in $\mathcal{D}^{\prime}(R)$ as

$$
(f(x), \phi(x))=\int_{-\infty}^{\infty} f(x) \phi(x) d x
$$

is well defined. Hence $f$ is linear and continuous on $\mathcal{D}(R)$. Furthermore, the functional $\delta\left(x-x_{0}\right)$ on $\mathcal{D}(R)$ given by

$$
\left(\delta\left(x-x_{0}\right), \phi(x)\right)=\phi\left(x_{0}\right)
$$

is a member of $\mathcal{D}^{\prime}(R)$, according to the topological structure of the Schwartz testing function space.

Let $f \in \mathcal{D}^{\prime}(R)$. The distributional derivative $f^{\prime}$ (or $d f / d x$ ), is defined as

$$
\left(f^{\prime}, \phi\right)=-\left(f, \phi^{\prime}\right)
$$

for $\phi \in \mathcal{D}(R)$. Therefore,

$$
\left(\delta^{(n)}\left(x-x_{0}\right), \phi(x)\right)=(-1)^{n}\left(\delta\left(x-x_{0}\right), \phi^{(n)}(x)\right)=(-1)^{n} \phi^{(n)}\left(x_{0}\right) .
$$

Let

$$
g(x)=x_{+}= \begin{cases}x & \text { if } x>0 \\ 0 & \text { otherwise }\end{cases}
$$

As an example, we will find the distributional derivative of $g$ (note that this function is not differentiable at $x=0$ in the classical sense). Indeed, using integration by parts, we derive

$$
\left(g^{\prime}(x), \phi(x)\right)=-\left(g(x), \phi^{\prime}(x)\right)=-\int_{0}^{\infty} x \phi^{\prime}(x) d x=\int_{0}^{\infty} \phi(x) d x=(\theta(x), \phi(x))
$$


which infers that

$$
g^{\prime}(x)=\theta(x)
$$

where $\theta(x)$ is the Heaviside function.

Let $\operatorname{Re} \lambda>-n-1, \lambda \neq-1,-2, \cdots,-n$. Then the distribution $x_{+}^{\lambda}$ [25] is defined by

$$
\begin{aligned}
\left(x_{+}^{\lambda}, \phi(x)\right)=\int_{0}^{\infty} x^{\lambda} \phi(x) d x= & \int_{0}^{1} x^{\lambda}\left[\phi(x)-\phi(0)-\cdots-\frac{t^{n-1}}{(n-1) !} \phi^{(n-1)}(0)\right] d t \\
& +\int_{1}^{\infty} x^{\lambda} \phi(x) d x+\sum_{k=1}^{n} \frac{\phi^{(k-1)}(0)}{(k-1) !(\lambda+k)} .
\end{aligned}
$$

Clearly, the right-hand side regularizes the integral on the left. This defines the distribution $x_{+}^{\lambda}$ for $\operatorname{Re} \lambda \neq-1,-2, \ldots$

Assume that $f$ and $g$ are distributions in $\mathcal{D}^{\prime}\left(R^{+}\right)$. Then the convolution $f * g$ is well defined by the equation [25]

$$
((f * g)(x), \phi(x))=(g(x),(f(y), \phi(x+y)))
$$

for $\phi \in \mathcal{D}(R)$. This also implies that

$$
f * g=g * f \text { and }(f * g)^{\prime}=g^{\prime} * f=g * f^{\prime} .
$$

Let $\mathcal{D}^{\prime}\left(R^{+}\right)$be the subspace of $\mathcal{D}^{\prime}(R)$ with support contained in $R^{+}$. It follows from [25-27] that $\Phi_{\lambda}=\frac{x_{+}^{\lambda-1}}{\Gamma(\lambda)} \in \mathcal{D}^{\prime}\left(R^{+}\right)$is an entire analytic function of $\lambda$ on the complex plane, and

$$
\left.\frac{x_{+}^{\lambda-1}}{\Gamma(\lambda)}\right|_{\lambda=-n}=\delta^{(n)}(x), \quad \text { for } n=0,1,2, \ldots
$$

which plays an important role in solving fractional differential equations by using the distributional convolutions. Let $\lambda$ and $\mu$ be arbitrary numbers, then the following identity

$$
\Phi_{\lambda} * \Phi_{\mu}=\Phi_{\lambda+\mu}
$$

is satisfied [23].

Let $\lambda$ be an arbitrary complex number and $g(x)$ be a distribution in $\mathcal{D}^{\prime}\left(R^{+}\right)$. We define the primitive of order $\lambda$ of $g$ as the distributional convolution

$$
g_{\lambda}(x)=g(x) * \frac{x_{+}^{\lambda-1}}{\Gamma(\lambda)}=g(x) * \Phi_{\lambda}
$$

Note that this is well defined since the distributions $g$ and $\Phi_{\lambda}$ are in $\mathcal{D}^{\prime}\left(R^{+}\right)$. We shall write the convolution

$$
g_{-\lambda}=\frac{d^{\lambda}}{d x^{\lambda}} g=g(x) * \Phi_{-\lambda}
$$

as the fractional derivative of the distribution $g$ of order $\lambda$ if $\operatorname{Re} \lambda \geq 0$, and $\frac{d^{\lambda}}{d x^{\lambda}} g$ is interpreted as the fractional integral if $\operatorname{Re} \lambda<0$.

We should add that Gorenflo and Mainardi [7] formally presented the derivative of order $n$ (non-negative integer) of $g$ by the generalized convolution between $\Phi_{-n}$ and $g$,

$$
\frac{d^{n}}{d x^{n}} g(x)=g^{(n)}(x)=\Phi_{-n}(x) * g(x)=\int_{0}^{x} g(\tau) \delta^{(n)}(x-\tau) d \tau
$$


based on the well known properties

$$
\int_{-\infty}^{\infty} g(\tau) \delta^{(n)}(\tau-x) d \tau=(-1)^{n} g^{(n)}(x), \quad \delta^{(n)}(x-\tau)=(-1)^{n} \delta^{(n)}(\tau-x) .
$$

Then, a formal definition of the fractional derivative of order $\lambda$ could be

$$
\Phi_{-\lambda}(x) * g(x)=\frac{1}{\Gamma(-\lambda)} \int_{0}^{x} \frac{g(\tau)}{(x-\tau)^{1+\lambda}} d \tau, \quad \lambda \in R^{+} .
$$

Note that this convolution is in the distributional sense (and it does not exist in the classical sense as the kernel $\Phi_{-\lambda}(x)$ is not locally integrable).

Example 1. Let

$$
g(x)= \begin{cases}1 & \text { if } 0<x<1 \text { and } x \text { is irrational } \\ 0 & \text { otherwise }\end{cases}
$$

Then,

$$
g^{(1.5)}(x)=\delta^{(0.5)}(x)-\delta^{(0.5)}(x-1)
$$

where

$$
\delta^{(0.5)}(x)=-\frac{x_{+}^{-1.5}}{2 \sqrt{\pi}} \quad \text { and } \quad \delta^{(0.5)}(x-1)=-\frac{(x-1)_{+}^{-1.5}}{2 \sqrt{\pi}} .
$$

In fact, we have for $\phi \in \mathcal{D}(R)$

$$
\begin{aligned}
\left(g^{\prime}(x), \phi(x)\right) & =-\left(g(x), \phi^{\prime}(x)\right)=-\int_{-\infty}^{\infty} g(x) \phi^{\prime}(x) d x=-\int_{0}^{1} \phi^{\prime}(x) d x \\
& =-\phi(1)+\phi(0)=(\delta(x)-\delta(x-1), \phi(x))
\end{aligned}
$$

as the measure of rational numbers is zero. This indicates that

$$
g^{\prime}(x)=\delta(x)-\delta(x-1)
$$

Obviously, by the sequential fractional derivative law, we come to

$$
g^{(1.5)}(x)=\frac{d^{0.5}}{d x^{0.5}} g^{\prime}(x)=\delta^{(0.5)}(x)-\delta^{(0.5)}(x-1)
$$

and

$$
\delta^{(0.5)}(x)=\frac{d^{0.5}}{d x^{0.5}} \delta(x)=\frac{d^{0.5}}{d x^{0.5}} \Phi_{0}=\frac{x_{+}^{-1.5}}{\Gamma(-0.5)}=-\frac{x_{+}^{-1.5}}{2 \sqrt{\pi}}
$$

using

$$
\Gamma(-0.5)=-2 \sqrt{\pi} .
$$

Furthermore,

$$
g^{(0.5)}(x)=\frac{d^{-0.5}}{d x^{-0.5}} g^{\prime}(x)=\delta^{(-0.5)}(x)-\delta^{(-0.5)}(x-1)
$$

where

$$
\begin{aligned}
& \delta^{(-0.5)}(x)=\Phi_{0.5} * \Phi_{0}=\Phi_{0.5}=\frac{x_{+}^{-0.5}}{\Gamma(0.5)}=\frac{x_{+}^{-0.5}}{\sqrt{\pi}} \\
& \delta^{(-0.5)}(x-1)=\frac{(x-1)_{+}^{-0.5}}{\sqrt{\pi}}
\end{aligned}
$$


which are regular distributions (locally integrable functions on $R$ ). In general, we can get

$$
g(x)=\theta(x)-\theta(x-1)
$$

distributionally by noting that

$$
\theta(x)=\Phi_{1}(x) .
$$

Then, we imply that for any complex number $\alpha \in C$

$$
\begin{aligned}
g^{(\alpha)}(x) & =\theta^{(\alpha)}(x)-\theta^{(\alpha)}(x-1)=\Phi_{-\alpha}(x) * \Phi_{1}(x)+\Phi_{-\alpha}(x) * \Phi_{1}(x-1) \\
& =\Phi_{1-\alpha}(x)+\Phi_{1-\alpha}(x-1)=\frac{x_{+}^{-\alpha}}{\Gamma(1-\alpha)}+\frac{(x-1)_{+}^{-\alpha}}{\Gamma(1-\alpha)} .
\end{aligned}
$$

In particular,

$$
g^{(5 / 2)}=\frac{3 x_{+}^{-5 / 2}}{4 \sqrt{\pi}}+\frac{3(x-1)_{+}^{-5 / 2}}{4 \sqrt{\pi}}
$$

by

$$
\Gamma(-3 / 2)=\frac{4}{3} \sqrt{\pi} .
$$

Now we are ready to present the following theorem.

Theorem 1. Let $g(x)$ be an infinitely differentiable function on $[0, \infty]$ and $f$ be an unknown distribution in $\mathcal{D}^{\prime}\left(R^{+}\right)$. Then the generalized Abel's integral equation of the first kind

$$
\theta\left(x-x_{0}\right) g(x)=\frac{1}{\Gamma(\alpha)} \int_{0}^{x}(x-\zeta)^{\alpha-1} f(\zeta) d \zeta
$$

has the solution

$$
f(x)=\theta\left(x-x_{0}\right) g(x) * \Phi_{-\alpha}
$$

where $\alpha$ is any real number in $R$ and $x_{0} \geq 0$. In particular, we have four different cases depending on the value of $\alpha$.

(i) If $m<\alpha<m+1$ for $m=0,1, \ldots$, then

$$
\begin{aligned}
f(x)= & \frac{d^{m+1}}{d x^{m+1}} \theta\left(x-x_{0}\right) g(x) * \frac{x_{+}^{-\alpha+m}}{\Gamma(-\alpha+m+1)} \\
= & g\left(x_{0}\right) \frac{\left(x-x_{0}\right)_{+}^{-\alpha}}{\Gamma(-\alpha+1)}+\cdots+g^{(m)}\left(x_{0}\right) \frac{\left(x-x_{0}\right)_{+}^{-\alpha+m}}{\Gamma(-\alpha+m+1)} \\
& +\frac{1}{\Gamma(-\alpha+m+1)} \int_{x_{0}}^{x} g^{(m+1)}(\zeta)(x-\zeta)^{-\alpha+m} d \zeta
\end{aligned}
$$

for $x \geq x_{0}$.

(ii) If $\alpha=1,2, \ldots$, then

$$
f(x)=g\left(x_{0}\right) \delta^{(\alpha-1)}\left(x-x_{0}\right)+\cdots+g^{(\alpha-1)}\left(x_{0}\right) \delta\left(x-x_{0}\right)+\theta\left(x-x_{0}\right) g^{(\alpha)}(x) .
$$

(iii) If $\alpha=0$, then $f(x)=\theta\left(x-x_{0}\right) g(x)$.

(iv) If $\alpha<0$, then for $x \geq x_{0}$

$$
f(x)=\frac{1}{\Gamma(-\alpha)} \int_{x_{0}}^{x} g(\zeta)(x-\zeta)^{-\alpha-1} d \zeta
$$

which is well defined. 
Proof. Clearly, we can convert Equation (4) into

$$
\theta\left(x-x_{0}\right) g(x)=f(x) * \Phi_{\alpha}
$$

which infers that

$$
\theta\left(x-x_{0}\right) g(x) * \Phi_{-\alpha}=\left(f(x) * \Phi_{\alpha}\right) * \Phi_{-\alpha}=f(x) * \Phi_{0}=f(x) * \delta(x)=f(x)
$$

by using Equations (1) and (2). Assuming $m<\alpha<m+1$ for $m=0,1, \ldots$, we derive that

$$
\Phi_{-\alpha}=\frac{d^{m+1}}{d x^{m+1}} \frac{x_{+}^{-\alpha+m}}{\Gamma(-\alpha+m+1)}
$$

where

$$
\frac{x_{+}^{-\alpha+m}}{\Gamma(-\alpha+m+1)}
$$

is a locally integrable function on $R$ since $-1<-\alpha+m<0$. Hence,

$$
f(x)=\theta\left(x-x_{0}\right) g(x) * \frac{d^{m+1}}{d x^{m+1}} \frac{x_{+}^{-\alpha+m}}{\Gamma(-\alpha+m+1)}=\frac{d^{m+1}}{d x^{m+1}} \theta\left(x-x_{0}\right) g(x) * \frac{x_{+}^{-\alpha+m}}{\Gamma(-\alpha+m+1)} .
$$

In particular, choosing $m=0$ (then $0<\alpha<1$ ) and $x_{0}=0$ we come to

$$
f(x)=\frac{1}{\Gamma(1-\alpha)} \int_{0}^{x} \frac{g^{\prime}(\zeta)}{(x-\zeta)^{\alpha}} d \zeta
$$

for a differentiable function $g(x)$ (in the classical sense). This is the solution for the classical Abel's integral equation.

Obviously, we get from integration by parts,

$$
\left(\frac{d}{d x} \theta\left(x-x_{0}\right) g(x), \phi(x)\right)=-\int_{x_{0}}^{\infty} g(x) \phi^{\prime}(x) d x=g\left(x_{0}\right) \phi\left(x_{0}\right)+\int_{x_{0}}^{\infty} \phi(x) g^{\prime}(x) d x
$$

where $\phi$ is the testing function. This implies that

$$
\frac{d}{d x} \theta\left(x-x_{0}\right) g(x)=g\left(x_{0}\right) \delta\left(x-x_{0}\right)+\theta\left(x-x_{0}\right) g^{\prime}(x) .
$$

By mathematical induction,

$$
\begin{aligned}
\frac{d^{m+1}}{d x^{m+1}} \theta\left(x-x_{0}\right) g(x) & =g\left(x_{0}\right) \delta^{(m)}\left(x-x_{0}\right)+g^{\prime}\left(x_{0}\right) \delta^{(m-1)}\left(x-x_{0}\right) \\
& =+\cdots+g^{(m)}\left(x_{0}\right) \delta\left(x-x_{0}\right)+\theta\left(x-x_{0}\right) g^{(m+1)}(x) .
\end{aligned}
$$

This infers that for $x \geq x_{0}$

$$
\begin{aligned}
f(x)= & \frac{d^{m+1}}{d x^{m+1}} \theta\left(x-x_{0}\right) g(x) * \frac{x_{+}^{-\alpha+m}}{\Gamma(-\alpha+m+1)} \\
= & g\left(x_{0}\right) \frac{\left(x-x_{0}\right)_{+}^{-\alpha}}{\Gamma(-\alpha+1)}+\cdots+g^{(m)}\left(x_{0}\right) \frac{\left(x-x_{0}\right)_{+}^{-\alpha+m}}{\Gamma(-\alpha+m+1)} \\
& +\frac{1}{\Gamma(-\alpha+m+1)} \int_{x_{0}}^{x} g^{(m+1)}(\zeta)(x-\zeta)^{-\alpha+m} d \zeta .
\end{aligned}
$$

The rest of the proof follows easily. This completes the proof of Theorem 1. 
Example 2. Let $m=0,1, \ldots$ Then the generalized Abel's integral equation

$$
\theta(x-1) e^{x}=\int_{0}^{x} f(\tau)(x-\tau)^{m-0.5} d \tau
$$

has the solution in $\mathcal{D}^{\prime}\left(R^{+}\right)$

$$
\begin{aligned}
f(x)= & \frac{1}{\left(\begin{array}{c}
m-0.5 \\
m
\end{array}\right) m ! \sqrt{\pi}}\left\{\delta^{(m-0.5)}(x-1)\right. \\
& \left.+\delta^{(m-3 / 2)}(x-1)+\cdots+\delta^{(-0.5)}(x-1)+\frac{1}{\sqrt{\pi}} \int_{1}^{x} \theta(\tau-1) e^{\tau}(x-\tau)^{-0.5} d \tau\right\} .
\end{aligned}
$$

Proof. Clearly, we can write Equation (5) to

$$
\theta(x-1) e^{x}=\frac{\Gamma(m+0.5)}{\Gamma(m+0.5)} \int_{0}^{x} f(\tau)(x-\tau)^{m-0.5} d \tau=\Gamma(m+0.5) \Phi_{m+0.5} * f,
$$

which deduces that by Theorem 1

$$
f(x)=\frac{1}{\Gamma(m+0.5)} \Phi_{-m-0.5} * \theta(x-1) e^{x}=\frac{1}{\Gamma(m+0.5)} \Phi_{0.5} * \Phi_{-m-1} * \theta(x-1) e^{x}
$$

as

$$
\Phi_{0} * f=\delta * f=f
$$

Obviously,

$$
\left(\frac{d}{d x} \theta(x-1) e^{x}, \phi(x)\right)=-\int_{1}^{\infty} e^{x} \phi^{\prime}(x) d x=\phi(1)+\int_{-\infty}^{\infty} \theta(x-1) e^{x} \phi(x) d x
$$

which claims that

$$
\frac{d}{d x} \theta(x-1) e^{x}=\delta(x-1)+\theta(x-1) e^{x}
$$

Similarly, we have

$$
\frac{d^{2}}{d x^{2}} \theta(x-1) e^{x}=\delta^{\prime}(x-1)+\delta(x-1)+\theta(x-1) e^{x} .
$$

By mathematical induction, we come to

$$
\frac{d^{m+1}}{d x^{m+1}} \theta(x-1) e^{x}=\delta^{(m)}(x-1)+\delta^{(m-1)}(x-1)+\cdots+\delta(x-1)+\theta(x-1) e^{x} .
$$

Therefore,

$$
\begin{aligned}
& \Phi_{0.5} * \Phi_{-m-1} * \theta(x-1) e^{x} \\
& =\Phi_{0.5} * \frac{d^{m+1}}{d x^{m+1}} \theta(x-1) e^{x} \\
& =\Phi_{0.5} *\left\{\delta^{(m)}(x-1)+\delta^{(m-1)}(x-1)+\cdots+\delta(x-1)+\theta(x-1) e^{x}\right\} \\
& =\delta^{(m-0.5)}(x-1)+\delta^{(m-3 / 2)}(x-1)+\cdots+\delta^{(-0.5)}(x-1)+\frac{1}{\sqrt{\pi}} \int_{1}^{x} \theta(\tau-1) e^{\tau}(x-\tau)^{-0.5} d \tau .
\end{aligned}
$$

This completes the proof by noting that

$$
\Gamma(m+0.5)=\left(\begin{array}{c}
m-0.5 \\
m
\end{array}\right) m ! \sqrt{\pi} .
$$


Example 3. Let $m=1,2, \ldots$ and

$$
\sin x_{+}= \begin{cases}\sin x & \text { if } x \geq 0 \\ 0, & \text { otherwise }\end{cases}
$$

Then the generalized Abel's integral equation

$$
\sin x_{+}=\int_{0}^{x} f(\tau)(x-\tau)^{-m-0.5} d \tau
$$

has the solution in $\mathcal{D}^{\prime}\left(R^{+}\right)$

$$
f(x)=\frac{\left(\begin{array}{c}
-0.5 \\
m
\end{array}\right) m ! x_{+}^{m+0.5}}{\sqrt{\pi}} E_{2, m+3 / 2}\left(-x^{2}\right)
$$

where $E_{\alpha, \beta}(x)$ is the Mittag-Leffler function, defined by the series expansion

$$
E_{\alpha, \beta}(x)=\sum_{k=0}^{\infty} \frac{x^{k}}{\Gamma(\alpha k+\beta)}
$$

for $\alpha, \beta>0$.

Proof. Evidently, we can convert Equation (6) to

$$
\sin x_{+}=\frac{\Gamma(-m+0.5)}{\Gamma(-m+0.5)} \int_{0}^{x} f(\tau)(x-\tau)^{-m-0.5} d \tau=\Gamma(-m+0.5) \Phi_{-m+0.5} * f
$$

Hence from Theorem 1, we get

$$
\begin{aligned}
f(x) & =\frac{1}{\Gamma(-m+0.5)} \Phi_{m-0.5} * \sin x_{+}=\frac{1}{\Gamma(-m+0.5) \Gamma(m-0.5)} \int_{0}^{x} \sin \tau(x-\tau)^{m-3 / 2} d \tau \\
& =\frac{1}{\Gamma(-m+0.5) \Gamma(m-0.5)} \sum_{k=0}^{\infty} \frac{(-1)^{k}}{(2 k+1) !} \int_{0}^{x} \tau^{2 k+1}(x-\tau)^{m-3 / 2} d \tau
\end{aligned}
$$

Setting $\tau=x t$, we arrive at

$$
\int_{0}^{x} \tau^{2 k+1}(x-\tau)^{m-3 / 2} d \tau=\frac{\Gamma(2 k+2) \Gamma(m-0.5)}{\Gamma(2 k+m+3 / 2)} x_{+}^{2 k+m+0.5} .
$$

This implies

$$
\begin{aligned}
f(x) & =\frac{1}{\Gamma(-m+0.5)} \sum_{k=0}^{\infty} \frac{(-1)^{k}}{\Gamma(2 k+m+3 / 2)} x_{+}^{2 k+m+0.5} \\
& =\frac{x_{+}^{m+0.5}}{\Gamma(-m+0.5)} \sum_{k=0}^{\infty} \frac{\left(-x^{2}\right)^{k}}{\Gamma(2 k+m+3 / 2)} \\
& =\frac{x_{+}^{m+0.5}}{\Gamma(-m+0.5)} E_{2, m+3 / 2}\left(-x^{2}\right)
\end{aligned}
$$

This completes the proof by using

$$
\Gamma(-m+0.5)=\frac{\sqrt{\pi}}{\left(\begin{array}{c}
-0.5 \\
m
\end{array}\right) m !}
$$


Furthermore, the generalized Abel's integral equation

$$
\delta^{(k)}(x)+x_{+}^{-\sqrt{3}}=\int_{0}^{x} f(\tau)(x-\tau)^{-m-0.5} d \tau
$$

has the solution in $\mathcal{D}^{\prime}\left(R^{+}\right)$

$$
f(x)=\frac{\left(\begin{array}{c}
-0.5 \\
m
\end{array}\right) m !}{\sqrt{\pi}} \Phi_{m-k-0.5}(x)+\frac{\left(\begin{array}{c}
-0.5 \\
m
\end{array}\right) \Gamma(-\sqrt{3}+1) m !}{\sqrt{\pi}} \Phi_{m-\sqrt{3}+0.5}(x)
$$

for $k=0,1, \ldots$ and $m=1,2, \ldots$.

Similarly, the generalized Abel's integral equation

$$
\delta^{(k)}(x)+x_{+}^{-\sqrt{3}}=\int_{0}^{x} f(\tau)(x-\tau)^{m-0.5} d \tau
$$

has the solution in $\mathcal{D}^{\prime}\left(R^{+}\right)$

$$
f(x)=\frac{1}{\left(\begin{array}{c}
m-0.5 \\
m
\end{array}\right) m ! \sqrt{\pi}} \Phi_{-m-k-0.5}(x)+\frac{\Gamma(-\sqrt{3}+1)}{\left(\begin{array}{c}
m-0.5 \\
m
\end{array}\right) m ! \sqrt{\pi}} \Phi_{-m-\sqrt{3}+0.5}(x) .
$$

for $k, m=0,1, \ldots$

To end off this section, we would add that many applied problems from physical, engineering and chemical processes lead to integral equations, which at first glance have nothing in common with Abel's integral equations, and due to this perception, additional efforts are undertaken for the development of analytical or numerical procedure for solving these equations. However, their transformations to the form of Abel's integral equations will speed up the solution process [24], or, more significantly, lead to distributional solutions in cases where classical ones do not exist [22].

As an example, the following integral equation with a moving integration limit

$$
\int_{0}^{y} \frac{1}{\left(y^{2}-x^{2}\right)^{\beta}} f(x) d x=g(y)
$$

can be converted into Abel's integral equation to solve in the distributional sense. The interested readers are referred to [22] for the detailed methods.

\section{Babenko's Approach in Distribution}

In this section, we shall extend the method used by Yu. I. Babenko in his book [28], for solving various types of fractional differential and integral equations in the classical sense, to generalized functions. The method itself is close to the Laplace transform method in the ordinary sense, but it can be used in more cases, such as solving fractional differential equations with variable coefficients. Clearly, it is always necessary to show convergence of the series obtained as solutions by other analytical tools, although it is a hard job in general [24]. We point out that Babenko's method can also be used to solve certain partial differential equations for heat and mass transfer theory, and suggest the interested readers are referred to [24] for the detailed arguments.

We must add that Oliver Heaviside (1850-1925) introduced an ingenious way of solving ordinary or partial differential equations arising from electromagnetic problems through algebration [29-31]. He considered, for example, the factor $d^{2} / d x^{2} f$ as product of the differential operator $d^{2} / d x^{2}$ with $f$ itself. Therefore, the differential equation for a given $g(x)$

$$
y(x)+y^{\prime \prime}(x)=g(x)
$$


can be converted to

$$
\left(1+d^{2} / d x^{2}\right) y(x)=g(x)
$$

which implies that the solution

$$
y(x)=\left(1+d^{2} / d x^{2}\right)^{-1} g(x)=\sum_{n=0}^{\infty}(-1)^{n} D^{2 n} g(x), \quad D=d / d x
$$

if it converges. This method is identical to Babenko's Approach.

Let $g(x) \in \mathcal{D}^{\prime}\left(R^{+}\right)$be given. We now study Abel's integral equation of the second kind

$$
y(x)+\frac{\lambda}{\Gamma(\alpha)} \int_{0}^{x}(x-\tau)^{\alpha-1} y(\tau) d \tau=g(x)
$$

with demonstrations of examples in the distributional space $\mathcal{D}^{\prime}\left(R^{+}\right)$, where $\alpha \in R$ and $\lambda$ is a constant. Further, we will investigate and solve several integral equations with variable coefficients by products of distributions and fractional operations of generalized functions. The results derived here cannot be achieved by the Laplace transform in general, or numerical analysis methods since distributions are undefined at points in $R$.

Clearly, Equation (8) is equivalent to the convolutional equation

$$
\left(\delta+\lambda \Phi_{\alpha}\right) * y(x)=g(x)
$$

in $\mathcal{D}^{\prime}\left(R^{+}\right)$, although it is undefined in the classical sense for $\alpha \leq 0$. We should point out it becomes the differential equation

$$
y+\lambda y * \delta^{(m)}=y+\lambda y^{(m)}=g
$$

if $\alpha=-m$.

Example 4. Let $\lambda$ be a nonzero constant and $\alpha>0$. Then the fractional differential equation

$$
\lambda y(x)+y^{(\alpha)}(x)=\delta(x)
$$

has the solution in the space $\mathcal{D}^{\prime}\left(R^{+}\right)$

$$
y(x)=x_{+}^{\alpha-1} E_{\alpha, \alpha}\left(-\lambda x_{+}^{\alpha}\right) .
$$

Proof. Equation (9) can be written into

$$
\lambda y+\Phi_{-\alpha} * y=\delta
$$

which is equivalent to

$$
\lambda \Phi_{\alpha} * y+y=\Phi_{\alpha} * \delta
$$

By Babenko's method we get

$$
\begin{aligned}
y(x) & =\sum_{n=0}^{\infty}(-1)^{n} \lambda^{n} \Phi_{\alpha}^{n+1} * \delta=\sum_{n=0}^{\infty}(-1)^{n} \lambda^{n} \Phi_{\alpha}^{n+1} \\
& =\sum_{n=0}^{\infty}(-1)^{n} \lambda^{n} \Phi_{\alpha(n+1)}=\sum_{n=0}^{\infty}(-1)^{n} \lambda^{n} \frac{x_{+}^{\alpha n+\alpha-1}}{\Gamma(\alpha n+\alpha)} \\
& =x_{+}^{\alpha-1} \sum_{n=0}^{\infty} \frac{\left(-\lambda x_{+}^{\alpha}\right)^{n}}{\Gamma(\alpha n+\alpha)}=x_{+}^{\alpha-1} E_{\alpha, \alpha}\left(-\lambda x_{+}^{\alpha}\right)
\end{aligned}
$$

which is convergent, and hence well defined, by noting that $x_{+}^{\alpha-1}$ is a locally integrable function on $R$. 
In particular, the differential equation

$$
\lambda y(x)+y^{\prime}(x)=\delta(x)
$$

has the solution in the space $\mathcal{D}^{\prime}\left(R^{+}\right)$

$$
y(x)=\theta(x) e^{-\lambda x} .
$$

Similarly, the fractional differential equation

$$
\lambda y(x)+y^{(\alpha)}(x)=x_{+}
$$

has the solution

$$
y(x)=x_{+}^{\alpha+1} E_{\alpha, \alpha+2}\left(-\lambda x_{+}^{\alpha}\right)
$$

where $\alpha>0$.

Indeed,

$$
\begin{aligned}
y(x) & =\sum_{n=0}^{\infty}(-1)^{n} \lambda^{n} \Phi_{\alpha}^{n+1} * x_{+}=\sum_{n=0}^{\infty}(-1)^{n} \lambda^{n} \Phi_{\alpha}^{n+1} * \frac{x_{+}}{\Gamma(2)} \\
& =\sum_{n=0}^{\infty}(-1)^{n} \lambda^{n} \Phi_{(n+1) \alpha} * \Phi_{2}=\sum_{n=0}^{\infty}(-1)^{n} \lambda^{n} \Phi_{(n+1) \alpha+2}(x) \\
& =x_{+}^{\alpha+1} \sum_{n=0}^{\infty}(-1)^{n} \lambda^{n} \frac{x_{+}^{\alpha n}}{\Gamma(n \alpha+\alpha+2)}=x_{+}^{\alpha+1} E_{\alpha, \alpha+2}\left(-\lambda x_{+}^{\alpha}\right) .
\end{aligned}
$$

Using the same argument, the fractional differential equation

$$
\lambda y(x)+y^{(\alpha)}(x)=\delta^{\prime}(x)
$$

has the solution

$$
y(x)=x_{+}^{\alpha-2} E_{\alpha, \alpha-1}\left(-\lambda x_{+}^{\alpha}\right)
$$

where $\alpha>1$ and $x_{+}^{\alpha-2}$ is a regular distribution.

More generally, the fractional differential equation

$$
y^{(\alpha)}(x)+\lambda y^{(\beta)}(x)=\delta(x), \quad \alpha>\beta \geq 0
$$

has the solution

$$
y(x)=x_{+}^{\alpha-1} E_{\alpha-\beta, \alpha}\left(-\lambda x_{+}^{\alpha-\beta}\right) .
$$

Remark 1. We begin by using Example 4 as a simple demonstration of Babenko's Approach. Clearly, all the equations presented above can be easily solved by the Laplace transform. For example, applying the Laplace transform to the equation

$$
y^{(\alpha)}(x)+\lambda y^{(\beta)}(x)=\delta(x), \quad \alpha>\beta \geq 0
$$

gives

$$
y^{*}(s)=\frac{1}{s^{\alpha}+\lambda s^{\beta}} .
$$

The inverse transform implies

$$
y(x)=x_{+}^{\alpha-1} E_{\alpha-\beta, \alpha}\left(-\lambda x_{+}^{\alpha-\beta}\right) .
$$


However, we must mention that Babenko's approach in the distributional sense is much more general than that of the Laplace transform. As an example, the Laplace transform does not work for the equation

$$
y^{(\alpha)}(x)+\lambda y^{(\beta)}(x)=x_{+}^{-1.5}, \quad \alpha>\beta \geq 0
$$

as the distribution $x_{+}^{-1.5}$ is not locally integrable. As indicated below, Babenko's approach provides an efficient method of dealing with this kind of equation.

Example 5. Let $\alpha>0$. Then the fractional differential and integral equation (mixed type)

$$
y^{(0.75)}(x)+\frac{\lambda}{\Gamma(\alpha)} \int_{0}^{x}(x-\tau)^{\alpha-1} y(\tau) d \tau=x_{+}^{-1.5}
$$

has the solution in the space $\mathcal{D}^{\prime}\left(R^{+}\right)$

$$
y(x)=-2 \sqrt{\pi} x_{+}^{-0.75} E_{\alpha+0.75,0.25}\left(-\lambda x_{+}^{\alpha+0.75}\right) .
$$

Proof. Equation (10) can be changed to

$$
y^{(0.75)}(x)+\lambda y^{(-\alpha)}(x)=\Gamma(-0.5) \Phi_{-0.5}(x)
$$

which is equivalent to

$$
\Phi_{-0.75} * y+\lambda \Phi_{\alpha} * y=-2 \sqrt{\pi} \Phi_{-0.5} .
$$

Applying $\Phi_{0.75}$ to both sides of the above equation, we only need to study the following integral equation

$$
y+\lambda \Phi_{\alpha+0.75} * y=-2 \sqrt{\pi} \Phi_{0.25}
$$

Therefore,

$$
\begin{aligned}
y(x) & =-2 \sqrt{\pi} \sum_{n=0}^{\infty}(-1)^{n} \lambda^{n} \Phi_{\alpha n+0.75 n} * \Phi_{0.25}=-2 \sqrt{\pi} \sum_{n=0}^{\infty}(-1)^{n} \lambda^{n} \Phi_{\alpha n+0.75 n+0.25} \\
& =-2 \sqrt{\pi} \sum_{n=0}^{\infty}(-1)^{n} \lambda^{n} \frac{x_{+}^{\alpha n+0.75 n-0.75}}{\Gamma(\alpha n+0.75 n+0.25)} \\
& =-2 \sqrt{\pi} x_{+}^{-0.75} \sum_{n=0}^{\infty} \frac{\left(-\lambda x_{+}^{\alpha+0.75}\right)^{n}}{\Gamma(\alpha n+0.75 n+0.25)} \\
& =-2 \sqrt{\pi} x_{+}^{-0.75} E_{\alpha+0.75,0.25}\left(-\lambda x_{+}^{\alpha+0.75)}\right.
\end{aligned}
$$

which is convergent.

Example 6. The generalized Abel's integral equation

$$
y(x)+\int_{0}^{x} \frac{y(\tau)}{\sqrt{x-\tau}} d \tau=x_{+}^{-\sqrt{2}}+\delta(x)
$$

has the solution in the space $\mathcal{D}^{\prime}\left(R^{+}\right)$

$$
\begin{aligned}
y(x)= & x_{+}^{-\sqrt{2}}+\delta(x)-x_{+}^{-0.5}-\frac{\Gamma(1-\sqrt{2}) \sqrt{\pi} x_{+}^{0.5-\sqrt{2}}}{\Gamma(3 / 2-\sqrt{2})}+\frac{\pi}{1-\sqrt{2}} x_{+}^{1-\sqrt{2}} \\
& -\Gamma(1-\sqrt{2}) \pi^{3 / 2} x_{+}^{3 / 2-\sqrt{2}} E_{0.5,5 / 2-\sqrt{2}}\left(-\sqrt{\pi} x_{+}^{0.5}\right)+\pi E_{0.5,1}\left(-\sqrt{\pi} t_{+}^{0.5}\right) .
\end{aligned}
$$


Proof. Equation (11) can be written into

$$
y(x)+\frac{\sqrt{\pi}}{\Gamma(0.5)} \int_{0}^{x} \frac{y(\tau)}{\sqrt{x-\tau}} d \tau=\Gamma(1-\sqrt{\pi}) \frac{x_{+}^{-\sqrt{2}}}{\Gamma(1-\sqrt{\pi})}+\frac{x_{+}^{-1}}{\Gamma(0)}=\Gamma(1-\sqrt{\pi}) \Phi_{1-\sqrt{\pi}}+\Phi_{0} .
$$

By Babenko's method we get

$$
\begin{aligned}
y(x)= & \sum_{n=0}^{\infty}(-1)^{n} \pi^{n / 2} \Phi_{n / 2} *\left(\Gamma(1-\sqrt{\pi}) \Phi_{1-\sqrt{\pi}}+\Phi_{0}\right) \\
= & \sum_{n=0}^{\infty}(-1)^{n} \pi^{n / 2} \Phi_{n / 2} * \Gamma(1-\sqrt{\pi}) \Phi_{1-\sqrt{\pi}} \\
& +\sum_{n=0}^{\infty}(-1)^{n} \pi^{n / 2} \Phi_{n / 2} \triangleq I_{1}+I_{2} .
\end{aligned}
$$

Clearly,

$$
\begin{aligned}
I_{2} & =\sum_{n=0}^{\infty}(-1)^{n} \pi^{n / 2} \Phi_{n / 2}=\sum_{n=0}^{\infty}(-1)^{n} \pi^{n / 2} \frac{x_{+}^{n / 2-1}}{\Gamma(n / 2)} \\
& =\delta(x)-\sqrt{\pi} \frac{x_{+}^{-0.5}}{\Gamma(0.5)}+\sum_{n=2}^{\infty}(-1)^{n} \pi^{n / 2} \frac{x_{+}^{n / 2-1}}{\Gamma(n / 2)} \\
& =\delta(x)-x_{+}^{-0.5}+\pi E_{0.5,1}\left(-\sqrt{\pi} x_{+}^{0.5}\right)
\end{aligned}
$$

where

$$
E_{0.5,1}(x)=e^{x^{2}} \operatorname{erfc}(-x)
$$

and $\operatorname{erfc}(x)$ is the error function complement defined by

$$
\operatorname{erfc}(x)=\frac{2}{\sqrt{\pi}} \int_{x}^{\infty} e^{-t^{2}} d t
$$

As for $I_{1}$, we come to

$$
\begin{aligned}
& I_{1}=\Gamma(1-\sqrt{2}) \sum_{n=0}^{\infty}(-1)^{n} \pi^{n / 2} \Phi_{n / 2+1-\sqrt{2}} \\
& =x_{+}^{-\sqrt{2}}-\frac{\Gamma(1-\sqrt{2}) \sqrt{\pi} x_{+}^{0.5-\sqrt{2}}}{\Gamma(3 / 2-\sqrt{2})} \\
& +\Gamma(1-\sqrt{2}) \pi \frac{x_{+}^{1-\sqrt{2}}}{\Gamma(2-\sqrt{2})}+\Gamma(1-\sqrt{2}) \sum_{n=3}^{\infty}(-1)^{n} \pi^{n / 2} \Phi_{n / 2+1-\sqrt{2}} \\
& =x_{+}^{-\sqrt{2}}-\frac{\Gamma(1-\sqrt{2}) \sqrt{\pi} x_{+}^{0.5-\sqrt{2}}}{\Gamma(3 / 2-\sqrt{2})}+\frac{\pi}{1-\sqrt{2}} x_{+}^{1-\sqrt{2}} \\
& -\Gamma(1-\sqrt{2}) \pi^{3 / 2} x_{+}^{3 / 2-\sqrt{2}} E_{0.5,5 / 2-\sqrt{2}}\left(-\sqrt{\pi} x_{+}^{0.5}\right) .
\end{aligned}
$$

The result follows from the sum of $I_{1}$ and $I_{2}$. 
Remark 2. Equation (11) cannot be discussed in the classical sense, including the Laplace transform, since the fractional integral or derivative of $x_{+}^{-\sqrt{2}}$, does not exist in the normal sense. Clearly, the distribution $x_{+}^{-\sqrt{2}}+\delta(x)$ in the solution is a singular generalized function in $\mathcal{D}^{\prime}\left(R^{+}\right)$, while the rest

$$
\begin{aligned}
& x_{+}^{-0.5}-\frac{\Gamma(1-\sqrt{2}) \sqrt{\pi} x_{+}^{0.5-\sqrt{2}}}{\Gamma(3 / 2-\sqrt{2})}+\frac{\pi}{1-\sqrt{2}} x_{+}^{1-\sqrt{2}} \\
& -\Gamma(1-\sqrt{2}) \pi^{3 / 2} x_{+}^{3 / 2-\sqrt{2}} E_{0.5,5 / 2-\sqrt{2}}\left(-\sqrt{\pi} x_{+}^{0.5}\right)+\pi E_{0.5,1}\left(-\sqrt{\pi} x_{+}^{0.5}\right) .
\end{aligned}
$$

is regular (locally integrable).

Assume $f$ is a distribution in $\mathcal{D}^{\prime}(R)$ and $g$ is a function in $C^{\infty}(R)$. Then the product $f g$ is well defined by

$$
(f g, \phi)=(f, g \phi)
$$

for all functions $\phi \in \mathcal{D}(R)$ as $g \phi \in \mathcal{D}(R)$. Therefore, the product, for $k=0,1, \ldots$,

$$
\frac{\lambda x^{k}}{\Gamma(\alpha)} \int_{0}^{x}(x-\tau)^{\alpha-1} y(\tau) d \tau
$$

makes sense since $x^{k}$ is in $C^{\infty}(R)$ and

$$
\frac{\lambda}{\Gamma(\alpha)} \int_{0}^{x}(x-\tau)^{\alpha-1} y(\tau) d \tau=\lambda \Phi_{\alpha} * y
$$

is a distribution in $\mathcal{D}^{\prime}\left(R^{+}\right)$(subspace of $\mathcal{D}^{\prime}(R)$ ) for arbitrary $\alpha \in R$ if $y \in \mathcal{D}^{\prime}\left(R^{+}\right)$, according to Section 2.

Example 7. Let $k \geq 1$ be an integer. Then the integral equation

$$
x^{k} \int_{0}^{x} \frac{y(\tau)}{\sqrt{x-\tau}} d \tau=\delta^{(m)}(x)
$$

has a solution in the space $\mathcal{D}^{\prime}\left(R^{+}\right)$

$$
y(x)=\frac{1}{\sqrt{\pi}}\left\{(-1)^{k} \frac{\delta^{(m+k+0.5)}(x)}{k !\left(\begin{array}{c}
m+k \\
k
\end{array}\right)}+C_{0} \delta^{(0.5)}(x)+\cdots+C_{k-1} \delta^{(k-0.5)}(x)\right\}
$$

where $m$ is a non-negative integer and $C_{0}, \cdots, C_{k-1}$ are arbitrary constants.

Proof. First, we show that

$$
x^{k} \delta^{(s)}(x)= \begin{cases}(-1)^{k} k !\left(\begin{array}{l}
s \\
k
\end{array}\right) \delta^{(s-k)}(x) & \text { if } k \leq s \\
0 & \text { otherwise }\end{cases}
$$

for $k, s=0,1,2, \ldots$

Indeed,

$$
\begin{aligned}
\left(x^{k} \delta^{(s)}(x), \phi(x)\right) & =\left.(-1)^{s}\left(x^{k} \phi(x)\right)^{(s)}\right|_{x=0} \\
& =(-1)^{s} k !\left(\begin{array}{l}
s \\
k
\end{array}\right) \phi^{(s-k)}(0) \\
& =(-1)^{k} k !\left(\begin{array}{l}
s \\
k
\end{array}\right)\left(\delta^{(s-k)}(x), \phi(x)\right)
\end{aligned}
$$

for $k \leq s$. 
On the other hand, we have

$$
x^{k} \delta^{(s)}(x)=0
$$

if $k>s$.

It follows that

$$
x^{k} \delta^{(m+k)}(x)=(-1)^{k} k !\left(\begin{array}{c}
m+k \\
k
\end{array}\right) \delta^{(m)}(x)
$$

Therefore,

$$
\int_{0}^{x} \frac{y(\tau)}{\sqrt{x-\tau}} d \tau=(-1)^{k} \frac{\delta^{(m+k)}(x)}{k !\left(\begin{array}{c}
m+k \\
k
\end{array}\right)}+C_{0} \delta(x)+\cdots+C_{k-1} \delta^{(k-1)}(x)
$$

which implies that

$$
\begin{aligned}
y(x) & =\frac{1}{\sqrt{\pi}} \Phi_{-0.5} *\left\{(-1)^{k} \frac{\delta^{(m+k)}(x)}{k !\left(\begin{array}{c}
m+k \\
k
\end{array}\right)}+C_{0} \delta(x)+\cdots+C_{k-1} \delta^{(k-1)}(x)\right\} \\
& =\frac{1}{\sqrt{\pi}}\left\{(-1)^{k} \frac{\delta^{(m+k+0.5)}(x)}{k !\left(\begin{array}{c}
m+k \\
k
\end{array}\right)}+C_{0} \delta^{(0.5)}(x)+\cdots+C_{k-1} \delta^{(k-0.5)}(x)\right\}
\end{aligned}
$$

where $C_{0}, \cdots, C_{k-1}$ are arbitrary constants.

Furthermore, we let $k \geq 1$ be an integer. Then the integral equation

$$
x^{k} \int_{0}^{x} \frac{y(\tau)}{(x-\tau)^{3 / 2}} d \tau=\delta^{(m)}(x)
$$

has a solution in the space $\mathcal{D}^{\prime}\left(R^{+}\right)$

$$
y(x)=-2 \sqrt{\pi}\left\{(-1)^{k} \frac{\delta^{(m+k-0.5)}(x)}{k !\left(\begin{array}{c}
m+k \\
k
\end{array}\right)}+C_{0} \delta^{(-0.5)}(x)+\cdots+C_{k-1} \delta^{(k-3 / 2)}(x)\right\}
$$

where $m$ is a non-negative integer and $C_{0}, \cdots, C_{k-1}$ are arbitrary constants.

In fact, we derive that

$$
\begin{aligned}
y(x) & =\Gamma(-0.5) \Phi_{0.5} *\left\{(-1)^{k} \frac{\delta^{(m+k)}(x)}{k !\left(\begin{array}{c}
m+k \\
k
\end{array}\right)}+C_{0} \delta(x)+\cdots+C_{k-1} \delta^{(k-1)}(x)\right\} \\
& =-2 \sqrt{\pi}\left\{(-1)^{k} \frac{\delta^{(m+k-0.5)}(x)}{k !\left(\begin{array}{c}
m+k \\
k
\end{array}\right)}+C_{0} \delta^{(-0.5)}(x)+\cdots+C_{k-1} \delta^{(k-3 / 2)}(x)\right\} .
\end{aligned}
$$

It generally follows that the integral equation

$$
x^{k} \int_{0}^{x} y(\tau)(x-\tau)^{\alpha-1} d \tau=\delta^{(m)}(x)
$$

has a solution

$$
y(x)=\frac{1}{\Gamma(\alpha)}\left\{(-1)^{k} \frac{\delta^{(m+k+\alpha)}(x)}{k !\left(\begin{array}{c}
m+k \\
k
\end{array}\right)}+C_{0} \delta^{(\alpha)}(x)+\cdots+C_{k-1} \delta^{(k+\alpha-1)}(x)\right\}
$$

where $\alpha \neq 0,-1, \ldots$ 
Example 8. Let $k \geq 1$ be an integer. Then the generalized integral equation

$$
x_{+}^{k} \int_{0}^{x} \frac{y(\tau)}{\sqrt{x-\tau}} d \tau=\delta^{(m)}(x)
$$

has a solution in the space $\mathcal{D}^{\prime}\left(R^{+}\right)$

$$
y(x)=\frac{1}{\sqrt{\pi}}\left\{\frac{2(-1)^{k} m !}{(m+k) !} \delta^{(k+m+0.5)}(x)+C_{0} \delta^{(0.5)}(x)+\cdots+C_{k-1} \delta^{(k-0.5)}(x)\right\} .
$$

where $m=0,1,2, \ldots$ and $C_{0}, \cdots, C_{k-1}$ are arbitrary constants.

Proof. To solve this integral equation, we require the following more complicated product of distributions, as $x_{+}^{k}$ is not an infinitely differentiable function (but it is a locally integrable function). It follows from Theorem 2.3 in [32] that

$$
x_{+}^{r} \delta^{(r+m)}(x)=\frac{(-1)^{r}(r+m) !}{2 m !} \delta^{(m)}(x)
$$

for $m, r=0,1,2, \ldots$. We note that this product can be derived directly from the complex analysis approach based on the Laurent series of $x_{+}^{\lambda}, x_{-}^{\lambda}$ as well as $e^{ \pm i \lambda \pi}$, given below

$$
\begin{aligned}
& x_{+}^{\lambda}=\frac{(-1)^{n-1}}{(n-1) !(\lambda+n)} \delta^{(n-1)}(x)+F_{-n}\left(x_{+}, \lambda\right), \\
& x_{-}^{\lambda}=\frac{1}{(n-1) !(\lambda+n)} \delta^{(n-1)}(x)+F_{-n}\left(x_{-}, \lambda\right), \\
& e^{ \pm i \lambda \pi}=(-1)^{n}[1 \pm(\lambda+n) \pi+\cdots] .
\end{aligned}
$$

Hence,

$$
\int_{0}^{x} \frac{y(\tau)}{\sqrt{x-\tau}} d \tau=\frac{2(-1)^{k} m !}{(m+k) !} \delta^{(k+m)}(x)+C_{0} \delta(x)+\cdots+C_{k-1} \delta^{(k-1)}(x)
$$

by noting that

$$
x_{+}^{k} \delta^{(i)}(x)=0
$$

where $i=0,1,2, \cdots, k-1$ and $C_{0}, \cdots, C_{k-1}$ are arbitrary constants. This implies that Equation (12) has a solution

$$
\begin{aligned}
y(x) & =\frac{1}{\sqrt{\pi}} \Phi_{-0.5} *\left\{\frac{2(-1)^{k} m !}{(m+k) !} \delta^{(k+m)}(x)+C_{0} \delta(x)+\cdots+C_{k-1} \delta^{(k-1)}(x)\right\} \\
& =\frac{1}{\sqrt{\pi}}\left\{\frac{2(-1)^{k} m !}{(m+k) !} \delta^{(k+m+0.5)}(x)+C_{0} \delta^{(0.5)}(x)+\cdots+C_{k-1} \delta^{(k-0.5)}(x)\right\} .
\end{aligned}
$$

Similarly, we let $k \geq 1$ be an integer. Then, the integral equation

$$
x_{+}^{k} \int_{0}^{x} \frac{y(\tau)}{(x-\tau)^{3 / 2}} d \tau=\delta^{(m)}(x)
$$

has a solution in the space $\mathcal{D}^{\prime}\left(R^{+}\right)$

$$
y(x)=-\frac{1}{2 \sqrt{\pi}}\left\{\frac{2(-1)^{k} m !}{(m+k) !} \delta^{(k+m-0.5)}(x)+C_{0} \delta^{(-0.5)}(x)+\cdots+C_{k-1} \delta^{(k-3 / 2)}(x)\right\}
$$


where $m$ is a non-negative integer, and $C_{0}, \cdots, C_{k-1}$ are arbitrary constants.

Clearly, the integral equation

$$
x_{+}^{k} \int_{0}^{x} y(\tau)(x-\tau)^{\alpha-1} d \tau=\delta^{(m)}(x)
$$

has a solution

$$
y(x)=\frac{1}{\Gamma(\alpha)}\left\{\frac{2(-1)^{k} m !}{(m+k) !} \delta^{(k+m+\alpha)}(x)+C_{0} \delta^{(\alpha)}(x)+\cdots+C_{k-1} \delta^{(k+\alpha-1)}(x)\right\}
$$

where $\alpha \neq 0,-1, \ldots$

Gorenflo and Mainardi [7] presented the applications of Abel's integral equations of the first and second kind to solve the following partial equation of heat flow:

$$
u_{t}-u_{x x}=0, \quad u=u(x, t)
$$

in the semi-infinite intervals $0<x<\infty$ and $0<t<\infty$ of space and time, respectively. Our results on the distributional Abel's integral equations have potential applications to dealing with differential equations in distribution and hence finding distributional (weak) solutions.

Remark 3. Generally speaking, there is the lack of definitions for nonlinear operations, such as product and composition in distribution theory, although it is of great demand in the areas of differential equations and quantum field theory. As an example, it seems hard to define the distribution $\delta^{2}(x)$, as

$$
\left(\delta^{2}(x), \phi(x)\right)=(\delta(x), \delta(x) \phi(x))=\delta(0) \phi(0)
$$

is undefined. Fisher, with his coauthors [33-41], has actively used the $\delta$-sequence and neutrix limit due to van der Corput since 1969, to deduce numerous products, powers, convolutions, and compositions of distributions by several workable definitions. From the above examples, it is clear to see the relations between products of generalized functions and integral equations with variable coefficients in distribution.

\section{Conclusions}

Applying Babenko's method and fractional calculus, we have studied and solved several fractional differential and integral equations, including ones with variable coefficients and a mixed type equation, in the distributional space $\mathcal{D}^{\prime}\left(R^{+}\right)$by using well defined products of generalized functions and the Mittag-Leffler function. In particular, we discussed Abel's integral equations of the first and second kind for arbitrary $\alpha \in R$ by fractional operations of distributions, and derived several new and interesting results which cannot be realized by numerical analysis, as they involve distributions which are undefined on $R$, or by the Laplace transform.

Author Contributions: The order of the author list reflects contributions to the paper.

Funding: This research was funded by NSERC (Canada) under grant number 2017-00001 and NSFC (China) under grant number 11671251.

Acknowledgments: The authors are grateful to the reviewers for the careful reading of the paper with several productive suggestions and corrections, which certainly improved its quality.

Conflicts of Interest: The authors declare no conflict of interest.

\section{References}

1. Zimbardo, G.; Perri, S.; Effenberger, F.; Fichtner, H. Fractional Parker equation for the transport of cosmic rays: Steady-state solutions. Astron. Astrophys. 2017, 607, A7. [CrossRef] 
2. Li, C.; Li, C.P. On defining the distributions $(\delta)^{k}$ and $\left(\delta^{\prime}\right)^{k}$ by fractional derivatives. Appl. Math. Comput. 2014, 246, 502-513.

3. Mann, W.R.; Wolf, F. Heat transfer between solids and gases under nonlinear boundary conditions. Quart. Appl. Math. 1951, 9, 163-184. [CrossRef]

4. Goncerzewicz, J.; Marcinkowska, H.; Okrasinski, W.; Tabisz, K. On percolation of water from a cylindrical reservoir into the surrounding soil. Appl. Math. 1978, 16, 249-261. [CrossRef]

5. Keller, J.J. Propagation of simple nonlinear waves in gas filled tubes with friction. Z. Angew. Math. Phys. 1981, 32, 170-181. [CrossRef]

6. Kilbas, A.A.; Srivastava, H.M.; Trujillo, J.J. Theory and Applications of Fractional Differential Equations; Elsevier: New York, NY, USA, 2006.

7. Gorenflo, R.; Mainardi, F. Fractional Calculus: Integral and Differential Equations of Fractional Order. In Fractals and Fractional Calculus in Continuum Mechanics; Carpinteri, A., Mainardi, F., Eds.; Springer: New York, NY, USA, 1997; pp. 223-276.

8. Wang, J.R.; Zhu, C.; Fečkan, M. Analysis of Abel-type nonlinear integral equations with weakly singular kernels. Bound. Value Probl. 2014, 2014, 20. [CrossRef]

9. Atkinson, K.E. An existence theorem for Abel integral equations. SIAM J. Math. Anal. 1974, 5, 729-736. [CrossRef]

10. Bushell, P.J.; Okrasinski, W. Nonlinear Volterra integral equations with convolution kernel. J. Lond. Math. Soc. 1990, 41, 503-510. [CrossRef]

11. Gorenflo, R.; Vessella, S. Abel Integral Equations: Analysis and Applications; Springer-Verlag: Berlin, Germany, 1991; Volume 1461.

12. Okrasinski, W. Nontrivial solutions to nonlinear Volterra integral equations. SIAM J. Math. Anal. 1991, 22, 1007-1015. [CrossRef]

13. Gripenberg, G. On the uniqueness of solutions of Volterra equations. J. Integral Equ. Appl. 1990, 2, 421-430. [CrossRef]

14. Mydlarczyk, W. The existence of nontrivial solutions of Volterra equations. Math. Scand. 1991, 68, 83-88. [CrossRef]

15. Kilbas, A.A.; Saigo, M. On solution of nonlinear Abel-Volterra integral equation. J. Math. Anal. Appl. 1999, 229, 41-60. [CrossRef]

16. Karapetyants, N.K.; Kilbas, A.A.; Saigo, M. Upper and lower bounds for solutions of nonlinear Volterra convolution integral equations with power nonlinearity. J. Integral Equ. Appl. 2000, 12, 421-448. [CrossRef]

17. Lima, P.; Diogo, T. Numerical solution of nonuniquely solvable Volterra integral equation using extrapolation methods. J. Comput. App. Math. 2002, 140, 537-557. [CrossRef]

18. Rahimy, M. Applications of fractional differential equations. Appl. Math. Sci. 2010, 4, 2453-2461.

19. Li, C.P.; Zeng, F. Numerical Methods for Fractional Calculus; Chapman and Hall/CRC: Boca Raton, FL, USA, 2015.

20. Mainardi, F. Fractional relaxation-oscillation and fractional diffusion-wave phenomena. Chaos Solitons Fractals 1996, 7, 1461-1477. [CrossRef]

21. Kesavan, S. Functional Analysis; Hindustan Book Agency: Gurgaon, India, 2014.

22. Li, C.; Li, C.P.; Kacsmar, B.; Lacroix, R.; Tilbury, K. The Abel integral equations in distributions. Adv. Anal. 2017, 2, 88-104. [CrossRef]

23. Li, C.; Clarkson, K. Babenko's approach to Abel's integral equations. Mathematics 2018, 6, 32. math6030032. [CrossRef]

24. Podlubny, I. Fractional Differential Equations; Academic Press: New York, NY, USA, 1999.

25. Gel'fand, I.M.; Shilov, G.E. Generalized Functions; Academic Press: New York, NY, USA, 1964; Volume I.

26. Li, C. Several results of fractional derivatives in $\mathcal{D}^{\prime}\left(R^{+}\right)$. Fract. Calc. Appl. Anal. 2015, 18, 192-207. [CrossRef]

27. Matignon, D. Stability results for fractional differential equations with applications to control processing. In Computational Engineering in System Applications; IMACS, IEEE-SMC: Lille, France, 1996; Volume 2, pp. 963-968.

28. Babenko, Y.I. Heat and Mass Transfer; Khimiya: Leningrad, Russia, 1986. (In Russian)

29. Heaviside, O. On operators in physical mathematics, Part 1. Proc. R. Soc. 1893, 52, 504-529. [CrossRef]

30. Heaviside, O. On operators in physical mathematics, Part 2. Proc. R. Soc. 1893, 54, 105-143. [CrossRef]

31. Heaviside, O. Electromagnetic Theory; Ernest Benn Ltd.: London, UK, 1925; Volumes 1, 2 and 3. 
32. Li, C. Several results on the commutative neutrix product of distributions. Integral Transforms Spec. Funct. 2007, 18, 559-568. [CrossRef]

33. Fisher, B.; Al-Sirehy, F. On the convolution and neutrix convolution of the functions $\sinh ^{-1} x$ and $x^{r}$. Sarajevo J. Math. 2015, 11, 37-48. [CrossRef]

34. Fisher, B.; Ozcag, E. Some results on the neutrix composition of the delta function. Filomat 2012, 26, 1247-1256. [CrossRef]

35. Fisher, B.; Ozcag, E.; Al-Sirehy, F. On the composition and neutrix composition of the delta function and the function $\cosh ^{-1}\left(|x|^{1 / r}+1\right)$. Int. J. Anal. Appl. 2017, 13, 161-169.

36. Fisher, B.; Taş, K. The convolution of functions and distributions. J. Math. Anal. Appl. 2005, 306, 364-374. [CrossRef]

37. Lazarova, L.; Jolevska-Tuneska, B.; Akturk, I.; Ozcag, E. Note on the distribution composition $\left(x_{+}^{\mu}\right)^{\lambda}$. Bull. Malaysian Math. Soc. 2016. [CrossRef]

38. Ozcag, E.; Lazarova, L.; Jolevska-Tuneska, B. Defining compositions of $x_{+}^{\mu},|x|^{\mu}, x^{-s}$ and $x^{-s} \ln |x|$ as neutrix limit of regular sequences. Commun. Math. Stat. 2016, 4, 63-80.

39. Li, C. The products on the unit sphere and even-dimension spaces. J. Math. Anal. Appl. 2005, 305, 97-106. [CrossRef]

40. Cheng, L.; Li, C. A commutative neutrix product of distributions on $R^{m}$. Math. Nachr. 1991, 151, $345-355$.

41. Li, C. A review on the products of distributions. In Mathematical Methods in Engineering; Taş, K., Tenreiro Machado, J.A., Baleanu, D., Eds.; Springer: Dordrecht, The Netherlands, 2007; pp. 71-96.

(C) 2018 by the authors. Licensee MDPI, Basel, Switzerland. This article is an open access article distributed under the terms and conditions of the Creative Commons Attribution (CC BY) license (http:/ / creativecommons.org/licenses/by/4.0/). 